\title{
Thiol-disulphide homeostasis is an oxidative stress indicator in critically ill children with sepsis
}

\author{
Ganime Ayar M.D, Sanliay Sahin ${ }^{b}$ M.D, Yasemin Men Atmaca $a^{a}$ M.D, \\ Mutlu Uysal Yazici M.D, Salim Neseliogluc M.D and Ozcan Erel ${ }^{c}$ M.D.
}

\section{ABSTRACT}

The aim of this study is to evaluate a novel oxidative stress marker (thiol-disulphide homeostasis) in paediatric sepsis and to determine their effects on the prognosis of sepsis. Patients diagnosed with sepsis $(\mathrm{n}=38)$ and healthy controls $(n=40)$ were incorporated in the study. Total thiol, native thiol, disulphide, disulphide/ total thiol, disulphide/ native thiol, and native thiol / total thiol levels were measured in the sepsis and control groups. Additionally, the parameters were compared between survivors and non-survivors in the sepsis group. The levels of hemoglobin, white blood cell, platelet, lactate, and C-reactive protein were measured in patients with sepsis at diagnosis. The paediatric risk of mortality and paediatric logistic organ dysfunction scores of the patients were used to estimate the disease severity. The plasma thiol levels of the patients with sepsis were significantly lower than the control group $(\mathrm{p}<0.001)$. This study showed that thiol/ disulphide homeostasis is abnormal in children with sepsis in Paediatric Intensive Care Unit. Key words: Sepsis, child, oxidative stress, thioldisulphide, homeostasis.

http:/ / dx.doi.org/10.5546/ aap.2019.eng.143 Hematology Oncology Training and Research Hospital.

b. University of Health Sciences, Dr. Sami Ulus Maternity and Children Training and Research Hospital.

c. University of Yildirim Beyazit, Ankara Atatürk Training and Research Hospital, Department of Biochemistry. Ankara, Turkey.

E-mail address:

Ganime Ayar, M.D.: ganimeayar@gmail.com

Funding:

None.

Conflict of interest: None.

Received: 8-10-2018 Accepted: 12-12-2018
To cite: Ayar G, Sahin S, Men Atmaca Y, Uysal Yazici M et al. Thiol-disulphide homeostasis is an oxidative stress indicator in critically ill children with sepsis. Arch Argent Pediatr 2019;117(3):143-148.

\section{INTRODUCTION}

Sepsis is a major worldwide cause of morbidity and mortality among infants and children. ${ }^{1,2}$ Mortality rate in paediatric sepsis ranges between $5 \%$ and $40 \%$ despite early recognition and aggressive early intervention for severe sepsis and septic shock. ${ }^{3}$ About $25 \%$ of deaths in Paediatric Intensive Care Unit (PICU)s are related to severe infections, and case fatality rates are as high as $17 \% .^{3}$ It has recently been reported that the incidence of severe infections in children that require admission to an intensive care unit (ICU) is increasing. ${ }^{4-6}$
Balance between the oxidants and the antioxidants in the organism is essential in maintaining cellular and biochemical functions. ${ }^{7}$ Oxidative stress (OS) causes cellular injury when the levels of reactive oxygen species increase above the physiological levels in the cells exposed to endogenous or exogenous oxidants. ${ }^{8}$ Oxidants damage the lipids, proteins, and DNA in the cells, and even lead to death. In patients dying from sepsis, light and electron microscopy as well as immunohistochemical staining for markers of cellular injury and stress revealed that cell death was rare in sepsis-induced cardiac and renal dysfunction. Moreover, the degree of cell injury or death did not account for severity of sepsis-induced organ dysfunction. ${ }^{9}$

Oxidative stress and antioxidant defence systems are also other factors with a role in the pathogenesis of sepsis. Studies have reported an increase in the amount of reactive oxygen species resulting from oxidative stress in patients diagnosed with sepsis. ${ }^{10}$ It has been reported that antioxidant potential might be low in sepsis patients at the beginning of the disease as compared to healthy subjects, but then begin to increase to normal levels and above., ${ }^{710}$

Plasma thiols are powerful antioxidants that physiologically eliminate the free radicals. Serum levels of protein thiols are among the indicators of antioxidant status in the body. Normally, there is a balance between the thiols and the disulphides and they also play a protective role in cellular redox homeostasis. This is called dynamic thiol/disulphide homeostasis. When this redox homeostasis tipped toward 
an overbalance of free radicals, then oxidative stress occurs. This process can be associated with various diseases. ${ }^{11,13}$

The present study aimed to investigate the thiol/ disulphide homeostasis which is of vital importance for anti-inflammatory and antioxidant processes in paediatric patients with sepsis and to explore their effects on the prognosis of sepsis.

\section{MATERIALS AND METHODS Study population}

This case-control study was conducted between March 2015 and February 2016. A total of 78 children (aged between 1 month and 18 years) participated in the study. The study group consisted of children who had been diagnosed with sepsis and followed in the University of Health Sciences, Ankara Child Health and Diseases Hematology Oncology Training and Research Hospital, Pediatric Intensive Care Unit (PICU), Ankara, Turkey.

The control group comprised age- and sexmatched children. Healthy children who were not diagnosed with any psychopathology and who were admitted to the department of pediatrics outpatient clinic because of routine checkups were selected for the control group.

Patients who were considered having sepsis according to "The Third International Consensus Definitions for Sepsis and Septic Shock (Sepsis-3)", ${ }^{14,15}$ based on their clinical and / or laboratory findings were included in the present study. These criteria includes the followings: 1) Two or more of: $a$. Temperature $>38^{\circ} \mathrm{C}$ or $<36{ }^{\circ} \mathrm{C} ; b$. heart rate $>90 / \mathrm{min} ; c$. respiratory rate $>20 / \mathrm{min}$ or $\mathrm{PaCO}_{2}<32 \mathrm{mmHg}(4,3 \mathrm{kPa})$; $d$. white blood cell count $>12000 / \mathrm{mm}$ or $<4000 /$ $\mathrm{mm}$ or $>10 \%$ immature bands, 2) Having at least one criteria; blood culture being positive or clinical sepsis (when blood culture was negative).

We excluded patients with the following criteria: age $<3$ months and $>18$ years, immunosuppressive, steroid, or radiation therapy, chronic diseases such as diabetes mellitus, chronic renal failure, rheumatologic diseases, oncologic patients and the use of any antioxidant agent (i.e., vitamins $E$ and $C$ ). The study was approved by the Local Ethics Committee and informed consent of the patients were obtained from the parents / caregivers of the patients.

\section{Procedures}

The levels of hemoglobin, WBC, platelet, lactate, total protein and CRP were measured in the blood samples drawn from the patients with sepsis at diagnosis or within the first 72 hours after diagnosis.

The paediatric risk of mortality (PRISM III) and paediatric logistic organ dysfunction (PELOD) scores of the patients were used to estimate the severity of disease.

Fasting blood samples were obtained from the patient and control groups and collected into plain tubes (BD Vacutainer ${ }^{\circledR}$, SST II Advance, REF 367955, UK) to measure antioxidants. Blood samples were centrifuged at $1500 \mathrm{~g}$ for 10 minutes within 20 minutes after drawing them from participants in order to obtain serum samples and then stored at $-80^{\circ} \mathrm{C}$ until analysis starts.

\section{Measurements}

\section{Laboratory method}

The data obtained were determined as total thiol, native thiol, disulphide, disulphide/total thiol, disulphide/native thiol, and native thiol/ total thiol levels.

Total thiol and native thiol concentrations in samples were measured using Ellmann's and modified Ellmann's reagent. Native thiol content was subtracted from total thiol content and half of this difference was calculated; this value was the amount of dynamic disulphide bonds. The ratios of disulphide / total thiol, disulphide/ native thiol, and native thiol/total thiol were calculated.

In this study, the tests of thiol/disulphide homeostasis were performed using the spectrophotometric method described by Erel et al. ${ }^{13}$ Homemade kits were applied the automated analyser Cobasc501 (Roche-Hitachi, Germany) according the described method.

\section{Statistical analysis}

Shapiro-Wilk test was used to check if continuous variables in the study such as age, total protein and total thiols were normally distributed. Descriptive statistics were expressed in numbers and percentages for categorical variables and in mean, standard deviation, median (IQR) for numerical variables. Normally distributed variables were expressed in mean \pm standard deviation $(\mathrm{M} \pm \mathrm{SD})$, nonnormally distributed variables were expressed in median and IQR (interquartile range percentile 25-75) and the categorical variables such as gender were expressed in percentages $(\%)$. Mann-Whitney U test was employed for two group comparisons of non-normally distributed numerical variables. Student's t-test was used for two group comparisons of normally distributed 
numerical variables. Statistical significance level was $p<0.05$. IBM SPSS Statistics for Windows version 21.0 (IBM Corp. Released 2012, Armonk, NY) programme was used for statistical analyses and calculations.

\section{RESULTS}

The sepsis group included 38 patients (17 girls/ 21 boys) with a median age of 3.75 years. The control group included 40 healthy children (20 girls/ 20 boys) with a median age of 4.75 years. No statistically significant difference was found for age between the controls and sepsis groups. Gender distribution was determined to be similar in both of the groups. Demographic and clinical characteristics of patients are provided in Table 1.
Distribution of values for native thiols, total thiols and disulphides by groups is given in Table 2. Native thiol, total thiol and disulphide levels were significantly lower $(p<0,001)$ in patients with sepsis compared to the control. Disulphide/ native thiol and disulphide/total thiol ratios were higher $(\mathrm{p}<0.05)$ in patients with sepsis.

The mortality rate of the patients diagnosed with sepsis was $28.9 \%$ (11 out of 38). Among the patients with sepsis, there were no significant differences between the survivors and nonsurvivors in terms of age, gender, and critical laboratory values (Table 3).

Comparison of thiol/disulphide homeostatic parameters at moment of sepsis diagnosis

TABLE 1. Distribution of age and gender among sepsis and control groups

\begin{tabular}{lccc}
\hline & Sepsis group $(\mathbf{n}=\mathbf{3 8})$ & Control group $(\mathbf{n}=\mathbf{4 0 )}$ & p-value \\
\hline Gender, $\mathbf{n}(\%)$ & $17(51.4)$ & $20(50.0)$ & 0.827 \\
Girl & $21(48.6)$ & $20(50.0)$ & 0.408 \\
Boy & $3.75(1.375-11.575)$ & $4.75(2.250-9.875)$ & \\
Age, years, median (IQR)
\end{tabular}

IQR: Interquartile range.

TABLE 2. Antioxidant levels in the sepsis and control groups

\begin{tabular}{|c|c|c|c|}
\hline & $\begin{array}{c}\text { Sepsis group } \\
(n=38)\end{array}$ & $\begin{array}{c}\text { Control group } \\
(n=40)\end{array}$ & p-value \\
\hline & Median (IQR) & Median (IQR) & \\
\hline Native thiol $(\mu \mathrm{mol} / \mathrm{L})$ & $260.75(220.9-332.0)$ & $451.40(431.93-463.95)$ & $<0.001$ \\
\hline Total thiol $(\mu \mathrm{mol} / \mathrm{L})$ & $284.25(241.53-361.15)$ & $483.85(465.98-504.33)$ & $<0.001$ \\
\hline Disulphide $(\mu \mathrm{mol} / \mathrm{L})$ & $13.48(10.24-17.63)$ & $19.43(15.26-21.64)$ & $<0.001$ \\
\hline Disulphide/Native thiol & $5,25(4.00-6.24)$ & $4.14(3.27-4.80)$ & 0.004 \\
\hline Disulphide/Total thiol & $4.75(3.59-5.55)$ & $3.82(3.13-4.38)$ & 0.006 \\
\hline Native thiol/Total thiol & 90.35 (88.14-91.96) & $92.32(91.23-93.75)$ & 0.001 \\
\hline
\end{tabular}

IQR: Interquartile range.

TABLE 3. Clinical and laboratory characteristics of survivors and non-survivors in the sepsis group

\begin{tabular}{|c|c|c|c|}
\hline & $\begin{array}{c}\text { Survivors } \\
(n=27)\end{array}$ & $\begin{array}{c}\text { Non-survivors } \\
\quad(n=11)\end{array}$ & p-value \\
\hline Gender & n $(\%)$ & n $(\%)$ & \\
\hline Girl & $14(52)$ & $4(36)$ & 0.392 \\
\hline \multirow[t]{2}{*}{ Boy } & $13(48)$ & $7(64)$ & \\
\hline & Median (IQR) & Median (IQR) & \\
\hline Age, years & $4.0(1.5-11.5)$ & $2.5(1.0-11.8)$ & 0.628 \\
\hline $\mathrm{WBC}, \times 10^{3} / \mu \mathrm{L}$ & $12.2(9.0-21.3)$ & $7.8(3.5-24.3)$ & 0.664 \\
\hline Hemoglobin, g/dL & $10.9(9.0-11.8)$ & $10.6(9.2-13.0)$ & 0.923 \\
\hline Platelet, $x 10^{3} / \mu \mathrm{L}$ & $193.0(51.0-315.0)$ & $136.0(24.0-367.0)$ & 0.987 \\
\hline $\mathrm{CRP}, \mathrm{mg} / \mathrm{dL}$ & $7.8(1.7-19.0)$ & $14.2(2.9-20.9)$ & 0.552 \\
\hline Lactate, $\mathrm{mmol} / \mathrm{L}$ & $2.1(1.4-3.9)$ & $2.7(1.7-4.2)$ & 0.384 \\
\hline \multirow[t]{2}{*}{ Total protein, $\mathrm{g} / \mathrm{dL}$} & $5.6(5.2-6.1)$ & $5.9(5.3-6.2)$ & 0.519 \\
\hline & Mean $( \pm S D)$ & Mean $( \pm S D)$ & \\
\hline Prism III, score & $14.89 \pm 9.39$ & $21,55 \pm 10.91$ & 0.067 \\
\hline PELOD, score & $18.96 \pm 13.02$ & $29.27 \pm 15.81$ & $<0.05$ \\
\hline
\end{tabular}

WBC: white blood cell; CRP: C-reactive protein; IQR: Interquartile range; SD: standard deviation. 
between surviving $(\mathrm{n}=27)$ and nonsurviving $(\mathrm{n}=11)$ are shown in Table 4. There was no significant difference between the survivor and non-survivor septic patients in terms of thiol values.

Additionally, no statistically significant correlation was determined between thiol / disulphide homeostatic parameters and prism and PELOD scores.

\section{DISCUSSION}

The pathogenesis of sepsis is a quite complex situation in which numerous mediators and various biomarkers play a role. The role of antioxidants in sepsis is based on limited number of studies conducted on adults..$^{8,9}$ Although thiol levels as an antioxidant parameter have been previously reported in other diseases, ${ }^{16-18}$ our current study is the first one investigating the dynamic thiol/disulphide homeostasis in children with sepsis. In this study, we aimed to determine the levels of dynamic thiol/disulphide homeostasis in paediatric patients with sepsis and to explore their effects on the prognosis. Dynamic thiol/disulphide homeostasis has critical roles in antioxidant protection as a novel marker of oxidative stress and is being increasingly implicated in many disorders; its determination provides valuable information on various normal or abnormal biochemical processes. ${ }^{11}$

We found that total thiol, native thiol and disulphide values, as well as native thiol/total thiol ratio, were lower in paediatric patients with sepsis. This indicates that antioxidant balance is impaired in patients with sepsis. These results were similar to those reported about antioxidant capacity that was previously studied in patients with sepsis and critical patients. ${ }^{19-21}$ In our study, disulphide/native thiol and disulphide/ total thiol ratios were higher in patients with sepsis. We attributed this to more reduction of native thiol and total thiol levels compared to disulphide levels.
Although there are limited number of studies on paediatric cases, antioxidant enzyme activities have been investigated in some adult studies. Kumar et al., ${ }^{19}$ determined increased levels of oxidants in septic adult patiens. Additionally, they also determined that antioxidant enzymes decrease in sepsis and, moreover, there was an inverse relationship between antioxidant enzymes and organ insufficiencies.

In a multi-center study conducted by Lorente et al., ${ }^{20}$ antioxidant capacity was measured in patients with sepsis and compared between nonsurvivors and survivors. They determined an association between total antioxidant capacity and mortality in severe sepsis and stated that

FIGURE 1. Patiens included flow chart

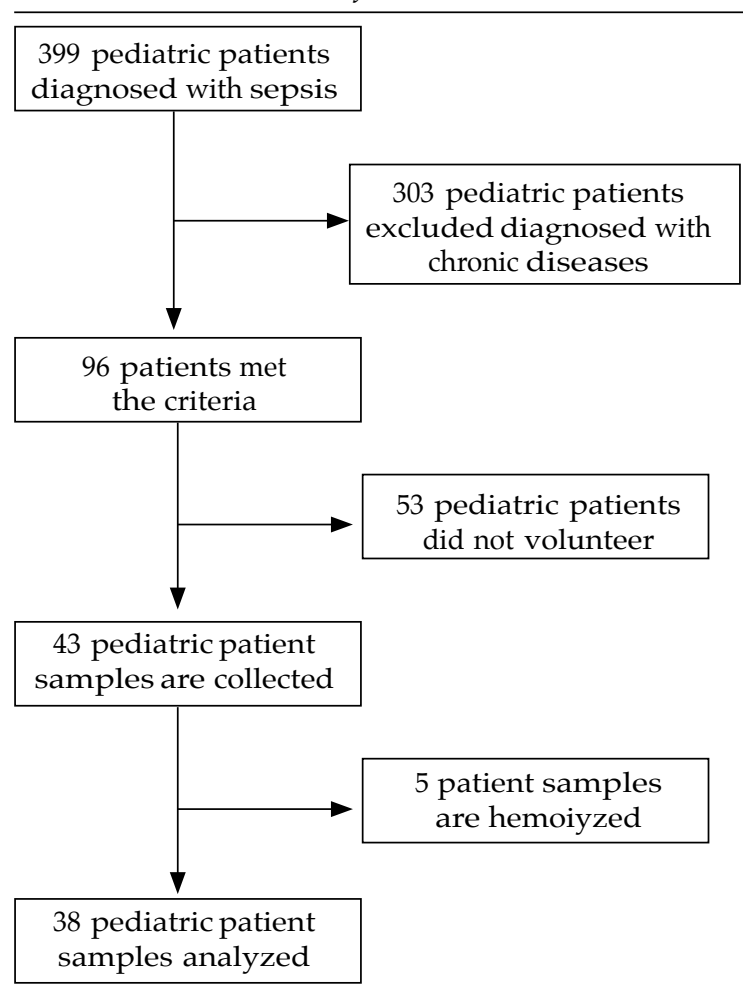

TABLE 4. Thiol/disulphide homeostatic parameters of survivors and non-survivors in the sepsis group

\begin{tabular}{lccc}
\hline Variable & Survivors $(\mathbf{n}=\mathbf{2 7})$ & Non-survivors $(\mathbf{n}=\mathbf{1 1})$ & $\mathbf{p}$ \\
\hline Native thiol $(\boldsymbol{\mu m o l} / \mathbf{L})$ & $268.4(197.7-332.9)$ & $249.9(230.0-342.2)$ & 0.834 \\
Total thiol $(\boldsymbol{\mu m o l} / \mathbf{L})$ & $296.4(223.3-358.1)$ & $275.5(254.8-371.0)$ & 0.961 \\
Disulphide $(\mu \mathrm{mol} / \mathbf{L})$ & $14.2(10.2-19.1)$ & $12.2(11.0-17.5)$ & 0.489 \\
Disulphide/native thiol & $5.4(4.1-6.7)$ & $5.1(3.1-5.6)$ & 0.253 \\
Disulphide/total thiol & $4.9(3.7-5.9)$ & $4.7(3.0-5.0)$ & 0.296 \\
Native thiol/total thiol & $89.8(87.8-91.9)$ & $90.7(89.9-94.1)$ & 0.119 \\
\hline
\end{tabular}

Parameters were expressed in median (interquartile range). 
antioxidant capacity was an indicator which predicts course of the disease in severe sepsis. In a recent study, ${ }^{21}$ they examined the antioxidant capacity during the first week in adult patients with sepsis and determined an association with severity of sepsis and mortality. Karapetsa et al., ${ }^{22}$ however, examined total antioxidant capacity on the first day of shock in patients with septic shock, which is an indicator of severe sepsis, and they could not find a significant difference between survivor and non-survivor patients and antioxidant balance. In consistence with the literature, PELOD score that reveals multiple organ dysfunction was high in our non-survivor patients. ${ }^{20,21}$

We know that antioxidant balance is important in patients with sepsis, especially in severe sepsis and concerning multiple organ dysfunction. It has been proposed that sepsisinduced organ dysfunction results at least partly from mitochondrial dysfunction caused by oxidative stress and this, in turn, results in energy insufficiency. ${ }^{23}$

In our study, we studied thiol/disulphide homeostasis in paediatric sepsis by a novel method and found it significantly lower in patients with sepsis compared to the control patients.

Limited number of cases, measuring antioxidant levels in a single blood sample and being single-centred are the limitations of the our study. Since there may be changes in antioxidant levels depending on the disease course and treatment, performing measurements in repetitive blood samples during follow-up period of the patients would give more information. In addition, we only looked at the determination of dynamic thiol/ disulphide homeostasis, which is just one part of the complex mechanism of oxidative stress. We could not determine the correlations among dynamic thiol/disulphide homeostasis and other oxidative parameters.

\section{CONCLUSIONS}

We showed that dynamic thiol/disulphide homeostasis was impaired in sepsis and thiol/ disulphide homeostasis is important in critically ill children. This novel method might be a simple tool which could be applied to routine screening as an indicator to show thiol-specific oxidative stress especially in paediatric sepsis. Nevertheless, further studies are required to support these findings.

\section{REFERENCES}

1. Randolph AG, McCulloh RJ. Pediatric sepsis: important considerations for diagnosing and managing severe infections in infants, children, and adolescents. Virulence. 2014; 5(1):179-89.

2. WheelerDS, Wong HR. Sepsis in Pediatric Cardiac Intensive Care. Pediatr Crit Care Med. 2016; 17(8 Suppl 1):S266-71.

3. de Souza DC, Barreira ER, Faria LS. The Epidemiology of Sepsis in Childhood. Shock. 2017; 47(Suppl 1):2-5.

4. Schlapbach LJ, Straney L, Alexander J, MacLaren G, et al. Mortality related to invasive infections, sepsis, and septic shock in critically ill children in Australia and New Zealand, 2002-13: a multi centre retrospective cohort study. Lancet Infect Dis. 2015; 15(1):46-54.

5. Hartman ME, Linde-Zwirble WT, Angus DC, Watson RS. Trends in the epidemiology of pediatric severe sepsis. Pediatr Crit Care Med. 2013; 14(7):686-93.

6. Balamuth F, Weiss SL, Neuman MI, Scott H, et al. Pediatric severe sepsis in U.S. children's hospitals. Pediatr Crit Care Med. 2014; 15(9):798-805.

7. Lorente L, Martín MM, Pérez-Cejas A, Abreu-González $P$, et al. Serum total antioxidant capacity during the first week of sepsis and mortality. J Crit Care. 2018; 47:139-44.

8. Harrois A, HuetO, DuranteauJ.Alterations of mitochondrial function in sepsis and critical illness. Curr Opin Anaesthesiol. 2009; 22(2):143-9.

9. Takasu O, GautJP, Watanabe E, To K, et al. Mechanisms of cardiac and renal dysfunction in patients dying of sepsis. Am J Respir Crit Care Med. 2013; 187(5):509-17.

10. Kumar S, Gupta E, Kaushik S, Kumar Srivastava V, Mehta SK, Jyoti A. Evaluation of oxidative stress and antioxidant status: Correlation with the severity of sepsis. Scand J Immunol. 2018; 87(4):e12653.

11. Davies KJ. Oxidative stress, antioxidant defenses, and damage removal, repair, and replacement systems. IUBMB Life. 2000; 50(4-5):279-89.

12. Haddad JJ. Oxygen sensing and oxidant/redox-related pathways. Biochem Biophys Res Commun. 2004;316(4):969-77.

13. ErelO, NeseliogluS. A novel and automated assay for thiol/ disulphide homeostasis. Clin Biochem. 2014; 47(18):326-32.

14. Singer M, Deutschman CS, Seymour CW, Shankar-Hari $\mathrm{M}$, et al. The Third International Consensus Definitions for Sepsis and Septic Shock (Sepsis-3). JAMA. 2016;315(8):80110.

15. Goldstein B, Giroir B, Randolph A; International Consensus Conference on Pediatric Sepsis. International pediatric sepsis consensus conference: definitions for sepsis and organ dysfunction in pediatrics. Pediatr Crit Care Med. 2005; 6(1):2-8.

16. Ozler S, Oztas E, Caglar AT, UygurD, et al. Thiol/disulfide homeostasis in predicting adverse perinatal outcomes at 24-28 weeks of pregnancy in gestational diabetes. J Matern Fetal Neonatal Med. 2016; 29(22):3699-704.

17. Kundi H, Ates I, Kiziltunc E, Cetin M, et al. A novel oxidative stress marker in acute myocardial infarction; thiol/disulphide homeostasis. Am J Emerg Med. 2015; 33(11):1567-71.

18. Ates I, Ozkayar N, Inan B, Yilmaz FM, et al. Dynamic thiol/ disulphide homeostasis in patients with newly diagnosed primary hypertension. J Am Soc Hypertens. 2016; 10(2):159-66.

19. Kumar S, Gupta E, Kaushik S, Kumar Srivastava V, et al. Evaluation of oxidative stress and antioxidant status: Correlation with the severity of sepsis. Scand J Immunol. 2018; 87(4):e12653.

20. Lorente L, Martín MM, Almeida T, Abreu-González P, et al. Association between serum total antioxidant capacity and mortality in severe septic patients. J Crit Care. 2015; 30(1):217.e7-12. 
148 / Arch Argent Pediatr 2019;117(3):143-148 / Original article

21. Lorente L, Martín MM, Pérez-Cejas A, Abreu-González $\mathrm{P}$, et al. Serum total antioxidant capacity during the first week of sepsis and mortality. J Crit Care. 2018; 47:139-44.

22. Karapetsa M, Pitsika M, Goutzourelas N, Stagos D, et al.
Oxidative status in ICU patients with septic shock. Food Chem Toxicol. 2013; 61:106-11.

23. Galley HF. Oxidative stress and mitochondrial dysfunction in sepsis. Br J Anaesth. 2011; 107(1):57-64. 